\title{
La tecnología móvil. Una herramienta para la mejora de la inclusión digital de las personas con TEA
}

\author{
Tecnologia móvel. \\ Uma ferramenta para melhorar a inclusão digital \\ de pessoas com TEA
}

\section{Mobile technology. A tool for improving the digital inclusion for people with ASD} \\ Mònica Sanromà-Giménez \\ Universitat Rovira i Virgili, España \\ José Luis Lázaro-Cantabrana \\ Universitat Rovira i Virgili, España \\ Mercè Gisbert-Cervera \\ Universitat Rovira i Virgili, España
}

Autor referente: monica.sanroma@urv.cat

Historia Editorial Recibido: 08/05/2017 Aceptado: 03/10/2017

\section{RESUMEN}

La digitalización de aspectos cotidianos de nuestra vida como el mundo laboral, el tiempo y espacio de ocio, la educación, la cultura, los medios de comunicación, entre muchos otros, nos ha generado la necesidad de

adaptarnos constantemente a los cambios producidos por la implantación de las tecnologías digitales. En el caso de las personas con trastorno del espectro autista (TEA), los avances evidenciados respecto a las 
tecnologías digitales y en especial de los dispositivos móviles han potenciado las posibilidades de desarrollarse $y$ formarse íntegramente como ciudadano activo así como mejorado su calidad de vida.

En los últimos años, la digitalización de los proceso de enseñanza y aprendizaje del alumnado con TEA se ha convertido en un campo de trabajo y estudio de interés científico generado por las evidencias recogidas respecto a sus múltiples beneficios. Por esta razón, actualmente existen numerosos recursos y apoyos tecnológicos para las diferentes áreas de intervención educativa con niños con TEA. Ante esta situación, es necesario evaluar los recursos y apoyos digitales desde una mirada crítica. Así como involucrar en este proceso de evaluación a los agentes educativos implicados.

Palabras clave: Tecnologías digitales; Inclusión digital; Trastorno del espectro autista; Tecnologías móviles

\section{RESUMO}

A digitalização de aspectos cotidianos de nossa vida como o mundo do trabalho, o tempo e espaços dedicados ao lazer, a educação, a cultura, os meios de comunicação, entre outros, gerou a necessidade de se adaptar constantemente às mudanças provocadas pela implantação das tecnologias digitais. No caso das pessoas com transtorno do espectro do autismo (TEA), os avanços realizados em relação às tecnologias digitais, especialmente dispositivos móveis, aumentaram as possibilidades de desenvolvimento e treinamento como cidadão ativo, além de melhorar sua qualidade de vida.
Nos últimos anos, a digitalização do processo de ensino e aprendizagem dos alunos com TEA tornou-se um campo de trabalho e pesquisa de interesse científico gerado pelas evidências coletadas em relação aos seus múltiplos benefícios. Por esta razão, existem atualmente muitos recursos e suporte tecnológico para as diferentes áreas de intervenção educacional com crianças com TEA. Dada esta situação, é necessário avaliar os recursos digitais e os apoios a partir de uma perspectiva crítica. Além de incluir neste processo de avaliação, os agentes educacionais envolvidos.

Palavras-chave: Tecnologias digitais; Inclusão digital; Desordem do espectro autista; Tecnologias móveis

\section{ABSTRACT}

The digitalisation of the diferent daily aspects of our life such as the world of work, leisure time and space, education, culture or media, among many other aspects, has brought the need to adapt constantly to the changes produced by the implantation of digital technologies. In the case of people with autism spectrum
disorder(ASD), the advances evidenced that have been made regarding to digital tecnologies and especially to Mobile devices have greatly favored their life and possibilities of developing and formin themselves as active citizens.

In the last years, the digitalisation of the teaching and learning process of 
students with ASD has become a field of work and study of scientific interest due to the gathered evidences of their múltiple benefits. For this reason, there are currently many resources and technological support for the diferent areas of educational intervention with children with ASD. Given this situation, it is necessary to evaluate digital resources and suports from a critical perspective. As well as engaging in this evaluation process the involved educational agents.

Key words: Digital tecnologies; Digital inclusion; Autism spectrum disorder; Mobile technologies

\section{Introducción}

W n este artículo nos proponemos recorrer algunas de las dimensiones de la inclusión digital de las personas con Trastorno del Espectro Autista (en adelante TEA) y el uso de las tecnologías digitales, en general, y los dispositivos móviles, en concreto, en los procesos de aprendizaje de este colectivo.

Pretendemos realizar una aproximación teórica del contexto social y educativo del que partimos: la constitución de nuevos espacios y oportunidades de aprendizaje motivados por la implantación de las tecnologías digitales, la selección y aplicación de estas para la atención educativa del alumnado con necesidades educativas específicas derivadas del TEA, cómo estas herramientas pueden favorecer su inclusión digital y, finalmente, el planteamiento de retos que podrían estimarse como relevantes para el futuro. Para ello hemos utilizado una metodología de revisión sistemática de los últimos diez años, explorando las aportaciones en publicaciones de impacto mediante bases de datos bibliográficas científicas (Web of Science y Scopus). Así indagamos desde un punto de vista crítico y fundamentado los siguientes puntos:

-La digitalización de la educación. Entre la brecha digital y la inclusión digital.

-El uso de dispositivos móviles para el desarrollo individualizado de los procesos de aprendizaje.

-El Trastorno del Espectro Autista (TEA). 
-Las tecnologías digitales como respuesta a las necesidades de las personas con TEA.

-Aplicaciones móviles para personas con TEA: ¿Cómo deben ser las Apps para personas autistas?

\section{La digitalización de la educación. Entre la brecha digital y la inclusión digital}

La presencia cada vez mayor de la tecnología en todas las dimensiones de nuestra vida cotidiana está generando grandes cambios en todos aquellos conceptos que hasta hace una década utilizábamos para poder definir y organizar los contextos educativos: el espacio (analógico y digital), el tiempo, la comunicación (síncrona y asíncrona) y la no diferenciación entre los diferentes ámbitos formativos (formal, no formal e informal). Estos cambios obligan a replantear los procesos de enseñanzaaprendizaje (E-A); desde la planificación hasta las estrategias de seguimiento y evaluación, pasando por la selección y la creación de los contenidos así como la selección y diseño de los medios y recursos (mayoritariamente digitales). En síntesis, todas las partes de este proceso se han visto modificadas, en un sentido u otro, a causa de la digitalización de la realidad educativa. El uso adecuado de los recursos tecnológicos en el ámbito educativo puede resultar de mucha utilidad a la hora de compensar las desigualdades provocadas por la rigidez del currículum, especialmente las de aquellas personas con necesidades específicas de apoyo educativo que están formándose tanto en el sistema educativo formal como en el ámbito no formal o informal (Lázaro, Estebanell\&Tedesco, 2015; Monclús \&Saban, 2012).

Compartimos con Monclús y Saban (2012) que la clave reside en la capacidad de evitar la exclusión mediante la creación de oportunidades para aquellos colectivos que 
se encuentran en situación de desventaja. Según la Fundación Orange (2014), en España, las personas con alguna discapacidad y las personas mayores de 55 años son aquellos colectivos con más riesgo de exclusión digital. En este sentido, será fundamental, tal como define la Agenda Digital Europea 2020, desarrollar estrategias políticas y sociales orientadas a favorecer, a través de la tecnología, la cohesión social. El desarrollo de acciones orientadas a democratizar el acceso a la información y al conocimiento, en todos los estratos y ámbitos sociales será fundamental para mejorar la calidad de vida de los colectivos menos favorecidos. También el uso de herramientas y estrategias que favorezcan tanto su desarrollo personal como su inclusión social, aumentando su bienestar, ayudará a reducir la brecha digital. Para que esta no se produzca y la tecnología no se convierta en una amenaza, se debe garantizar su acceso abierto y para todos. Además de dinamizar el entorno, las tecnologías deben permitir detectar las necesidades educativas y sociales, orientar las políticas públicas y promover acciones encaminadas a la adquisición de conocimiento y acceso a la sociedad digital en igualdad de condiciones. Si no facilitamos el acceso a los recursos tecnológicos a toda la ciudadanía, de manera universal, las personas que no puedan acceder a ellos se verán claramente marginadas (Rivoir, 2009). La generalización del uso de la tecnología por parte de la sociedad actual nos debe hacer considerar cuales son los efectos y sus consecuencias en todo aquello relacionado con la inclusión social y la posibilidad de participar de una sociedad cada vez más digitalizada provocando, como acabamos de mencionar, la brecha digital. Con el fin de compensar los efectos de exclusión derivados de esta, es necesario considerar las siguientes dos estrategias. En primer lugar, las políticas gubernamentales orientadas a facilitar el acceso de la ciudadanía a la tecnología (recursos materiales e infraestructuras), como por ejemplo el Plan Ceibal 
implementado en Uruguay o la iniciativa de CILSA mediante el Programa Nacional de Becas y Oportunidades orientado a la formación de colectivos en situación de vulnerabilidad del gobierno de Argentina. En segundo lugar, el seguimiento y la evaluación de las políticas impulsadas (Lázaro et al., 2015). A continuación, presentamos una serie de principios propuestos por Picard y Pickard (2017) para garantizar la inclusión en una sociedad digital:

- Satisfacer las necesidades fundamentales en comunicación y contenidos.

- Proveer a las personas de las habilidades necesarias para poder hacer un uso efectivo de los medios y las comunicaciones.

- Proporcionar diversidad y pluralidad en la propiedad de los medios y contenidos disponibles.

- Proteger a los usuarios y a la sociedad.

- Promover la transparencia y la responsabilidad.

- Perseguir el desarrollo y los beneficios económicos.

- Perseguir una política equitativa y efectiva.

Haciendo referencia al segundo principio, es necesario asegurar la competencia digital de los ciudadanos, sin distinción de estratos sociales ni de capacidades personales, fomentado el uso de las tecnologías digitales. Para ello es necesario que existan suficientes canales que faciliten la compartición de la información y los contenidos digitales. En realidad, como más dificultades presente la persona, por razones económicas, sociales, personales, etc. a la hora de desarrollar la competencia digital, más atención deberemos prestarle en términos educativos. Este es el caso de todas aquellas personas que sufren algún tipo de alteración que les impide seguir el ritmo de desarrollo educativo considerado ordinario. 
Para ello, es necesario llevar a cabo acciones formativas, no solo en el ámbito formal, sino también en el no formal a la vez que asegurar el acceso abierto a todos los recursos digitales que puedan favorecer al aprendizaje informal tanto desde la perspectiva individual como desde la colectiva. Simultáneamente, hemos de asumir la responsabilidad, como educadores, de garantizar un nivel de competencia digital adecuado a todos los integrantes de la comunidad educativa considerando, especialmente al ámbito familiar. Esta comunidad debe ser capaz de integrar y usar las tecnologías digitales para el desarrollo del proceso educativo con cualquier perfil de aprendiz y con independencia de las necesidades de aprendizaje específicas de este aunque teniéndolas en cuenta a la hora de seleccionarlas.

La UNESCO (2014) destaca que las herramientas tecnológicas favorecen la accesibilidad, la autonomía, el acceso a la educación, en general, y el acceso al aprendizaje, en particular, a la participación, a la comunicación, a la información, a la movilidad y a la adaptación al medio. Herramientas que ya las podemos llevar en el bolsillo y que su portabilidad favorece su uso en todo momento y en cualquier contexto educativo. Sea cuál sea la situación del aprendiz, sus necesidades y características éste puede disponer de dispositivos fáciles de manejar, que están conectados a la red y por tanto permiten el acceso a infinidad de información, contenidos y recursos. Los dispositivos móviles, desde la perspectiva de la personalización de los procesos de aprendizaje, han supuesto un gran avance, especialmente en aquellos casos que el sujeto tiene problemas de expresión y de comunicación.

En este sentido, y para poder garantizar el uso adecuado de los dispositivos móviles, es fundamental disponer de docentes bien formados, capaces de utilizar el potencial de las tecnologías digitales para atender a la diversidad de necesidades educativas de sus alumnos. También para formarlos en competencia digital para que sean capaces 
de participar de forma efectiva en una sociedad en la que las tecnologías digitales forman parte de la cotidianidad. Al hablar de participación nos referimos a un concepto amplio del mismo que va más allá del desarrollo de las capacidades como individuo, incluyendo la interacción con el contexto social, político, cultural e institucional.

\section{El uso de dispositivos móviles para el desarrollo individualizado de los} procesos de aprendizaje

Los dispositivos móviles son aquellas herramientas que nos facilitan el acceso permanente y constante a la información y a la comunicación. Por esta razón, en condiciones óptimas de conectividad y personales pueden convertirse en un apoyo fundamental en el proceso de acceso y construcción del conocimiento. Esta tecnología, aplicada de forma correcta en los procesos educativos puede enriquecer la comunicación y construcción de conocimiento de cualquier aprendiz independientemente de sus capacidades y limitaciones. Debemos, para ello, conocer las potencialidades de los dispositivos móviles y utilizarlos en aquellos momentos del proceso que más nos pueden ayudar a conseguir nuestros objetivos educativos.

El uso masivo de los dispositivos móviles es considerado una evidencia de la impregnación de las tecnologías digitales en todos los ámbitos de la sociedad. En la última década, la progresión en el uso de estos dispositivos ha crecido exponencialmente, llegando a alcanzar los 7,9 millones, más que habitantes hay en nuestro planeta. En concreto, al referirnos al uso de teléfonos móviles, se prevé que en 2020 el $80 \%$ de la población mundial dispondrá de ellos, siendo en Latinoamérica donde se experimentará un crecimiento mayor (Cisco, 2016). Los teléfonos inteligentes o smartphones son los que presentan un mayor crecimiento en los últimos años. Así, por ejemplo, en el año 2016 en España el 80\% de la población disponía de 
uno de ellos, llegando a superar el número de ordenadores por usuario (Ditrendia, 2016).

Los móviles, las tabletas, los lectores-e, los reproductores de audio portátiles, las consolas de videojuegos, los miniordenadores, entre otros, son herramientas que deberíamos incorporar en los procesos de enseñanza-aprendizaje (E-A) puesto que nos pueden ayudar a mejorarlo en cualquier momento y en cualquier lugar a la vez que pueden constituir un mecanismo importante de innovación educativa (Gisbert, Prats \& Cabrera, 2015).

Cada vez más, los dispositivos móviles están sustituyendo a los ordenadores para conectarse a Internet. A modo de ejemplo, según el informe anual de Telefónica (2016), en España, el 88,3\% de los internautas prefieren el smartphone como principal dispositivo para conectarse a la red.

Las explicaciones a este fenómeno se asocian a la gran expansión de las aplicaciones móviles (en adelante Apps) para estos dispositivos. También al concepto de "tecnologías de bolsillo". La realidad es que estas permiten entre muchas otras cosas, estar conectado mientras se realizan actividades cotidianas, acceder de forma inmediata a los recursos disponibles en la red, así como fusionar la vida real y la digital mediante una conexión inmediata y disponible en cualquier lugar (Gisbert, Prats \& Cabrera, 2015; Telefónica, 2016).

\subsection{Las tecnologías digitales para atender las necesidades educativas}

\section{específicas}

Las tecnologías digitales se han convertido en herramientas de apoyo verdaderamente útiles y en un potente recurso para las personas con necesidades educativas específicas (en adelante NEE) en varios ámbitos: educación, comunicación, ocio y 
tiempo libre, valoración y diagnóstico, etc. De todos ellos cabe destacar en concreto el ámbito de la educación y la comunicación, donde las tecnologías digitales se hacen imprescindibles especialmente para las personas con TEA. Este trastorno engloba un conjunto heterogéneo de alteraciones del desarrollo neuronal que incluye, además de un trastorno del desarrollo socio-comunicativo y un patrón restringido de intereses y conductas repetitivas, otro tipo de manifestaciones clínicas que varían enormemente de un individuo a otro (Asociación Americana de Psiquiatría, 2013).

Los recursos tecnológicos diseñados para la intervención directa con personas con alguna necesidad de aprendizaje específica y especialmente con TEA han evolucionado mucho en los últimos años. Inicialmente el uso de la tecnología se concretaba en la utilización de recursos como vídeos y dispositivos fijos como ordenadores de sobremesa o portátiles, limitando así las posibilidades de interacción. Actualmente el uso de la tecnología digital se ha extendido exponencialmente llegando a campos tan versátiles como la robótica y la realidad virtual (Kientz, Goodwin, Hayes \& Abowd, 2013).

De ahí que las tecnologías digitales puedan llegar a tener un papel muy importante en la medida en que se sepa intervenir con ellas pues ofrecen una gran cantidad de recursos didácticos, situaciones y escenarios de aprendizaje más significativos y motivadores que los tradicionales.

Con el fin de comprender y explorar más detalladamente las potencialidades de las tecnologías digitales y en concreto las Apps en la intervención educativa con personas con TEA, consideramos apropiado introducir conceptualmente dicho trastorno. Nos gustaría puntualizar que en estos últimos años las investigaciones sobre el TEA, realizadas des de diferentes disciplinas, han experimentado un avance importante que 
ha permitido acercarnos más a la comprensión de un trastorno del neurodesarrollo tan complejo.

\section{El Trastorno del Espectro Autista (TEA)}

\subsection{Definición, causas y prevalencia}

El concepto de TEA es reciente, anteriormente se utilizaba el término autismo infantil considerándose un trastorno raro de la infancia asociado a la ausencia de conciencia social, dificultades en el lenguaje y discapacidad intelectual grave.

Actualmente, el término TEA hace referencia a un conjunto amplio de condiciones que afectan al neurodesarrollo y al funcionamiento cerebral, dando lugar a dificultades en la comunicación e interacción social, así como en la flexibilidad del pensamiento y de la conducta (APA, 2013).

Hay personas en las cuales el trastorno se acompaña de una discapacidad intelectual, condiciones psiquiátricas concretas, otras muestran una alteración grave en el desarrollo lingüístico, y otras, a pesar de compartir este mismo diagnóstico, presentan un alto potencial cognitivo o habilidades lingüísticas. Esta variabilidad no solo se observa de una persona a otra, sino también a lo largo del ciclo vital de una misma.

Los criterios diagnósticos del TEA han evolucionado a lo largo de las diferentes publicaciones del Manual Diagnóstico y Estadístico de Trastornos Mentales (DSM) aunque seguimos desconociendo los marcadores biológicos que ayudan a diagnosticarlo. Actualmente el TEA se diagnostica según marcadores conductuales, es decir, según las conductas observables en la persona.

En el DSM-5 (APA, 2013) las personas diagnosticadas con TEA se caracterizan por mostrar dificultades en únicamente dos áreas: (1) el área socio-comunicativa y (2) el 
área de los intereses fijos y conductas repetitivas. También se definen tres grados de severidad según las dificultades que presenta el niño o niña en estas dos áreas del desarrollo.

Hoy en día, no se conoce con exactitud los factores que provocan el TEA a pesar de que se señale firmemente hacia componentes genéticos y factores ambientales.

En cuanto a la prevalencia se ha evidenciado que en los últimos años se ha constatado un aumento considerable de los casos detectados y diagnosticados (Hansen, Schendel \& Parner, 2015). Es posible que este incremento se deba a una mayor precisión de los procedimientos, criterios e instrumentos de diagnóstico, a la mejora en el conocimiento y la formación de los profesionales o a un aumento real de la incidencia de este tipo de trastornos.

Datos publicados por el Centers for Disease Control and Prevention (CDC, 2015) estiman que:

- A través de estudios realizados en Asia, Europa y América del Norte se conoce que la prevalencia media de personas diagnosticadas con TEA se sitúa entre el $1 \%$ y $2 \%$.

- 1 de cada 68 niños fueron diagnosticados con TEA en Estados Unidos.

- EI TEA es más común entre los varones que entre las niñas.

\subsection{Características y sintomatología}

EI TEA es un trastorno muy complicado de clasificar y diagnosticar pero normalmente se identifican señales de alerta y rasgos característicos en los primeros momentos del desarrollo del niño, entre los 18 y 24 meses de edad aproximadamente. Según Riviére (2002) estos son algunos de los rasgos característicos más evidentes. 
Tabla 1: Rasgos característicos del autismo

\section{Aparición de dificultades de interacción social}

- Desarrollo de habilidades de referencia conjunta, es decir, al compartir acciones e intereses: no muestran los objetos que les resultan interesantes, no imitan o repiten gestos, no comparten la atención con otras personas.

- Uso inadecuado de conductas no verbales para la regulación de la interacción social: expresiones faciales limitadas e inusuales, ausencia de respuesta no verbal al contacto físico, no mantienen la mirada.

- Integración escasa de la mirada en otros comportamientos para comunicar intención social: no mirar a los ojos.

- Fracaso en el desarrollo de relaciones con los iguales, adecuadas a su edad.

- Dificultades para comprender el comportamiento de las otras personas.

- Interacción inadecuada con otros niños, por ejemplo juegos físicos bruscos.

- Falta de adecuación con las normas sociales.

\section{Aparición de dificultades en el lenguaje y la comunicación}

- Sordera aparente: no responden cuando se les llama por su nombre.

- Uso de gestos muy limitados, solo para señalar necesidades básicas.

- Retraso en el desarrollo del lenguaje o retrocesos en la adquisición del lenguaje.

- Poco uso del lenguaje no verbal como la mímica y la gesticulación.

- Alteraciones en el uso del lenguaje aunque este sea fluido.

- Uso estereotipado y repetitivo del lenguaje, ecolalias.

- Comprensión literal del lenguaje, no se distingue el lenguaje literal y el figurado.

- Uso de neologismos.

Aparición de dificultades en la flexibilidad y la imaginación 
- Carencias en el desarrollo del juego creativo y simbólico, solo aparece el juego repetitivo.

- En el juego, presentan fijación en las partes o detalles inusuales de los juguetes.

- Resistencia al cambio, causando ansiedad e inseguridad.

- Frecuencia de movimientos estereotipados.

Nota. Adaptación de Rivière (2002).

Las especiales características de las personas con TEA deberán tenerse en cuenta en el diseño, desarrollo y evaluación de los recursos educativos digitales que se utilicen con ellas.

\section{Las tecnologías digitales como respuesta a las necesidades de las personas con TEA}

La mayoría de niños se sienten atraídos por la tecnología, pero los niños con TEA la encuentran mucho más atractiva debido a la importancia que para ellos tiene toda aquella información recibida visualmente en el procesamiento de la información. Estudios como los realizados por Parsons, Leonard y Mitchell (2006) demuestran que las actividades presentadas a través de un entorno o recurso digital pueden motivar y predisponer más el aprendizaje de los niños con TEA porque se trabaja más con estímulos multisensoriales (preferentemente visuales) provocando que aprenda disfrutando.

Las tecnologías digitales además de estar disponibles fácilmente y ser motivadoras, promueven el desarrollo de actividades colaborativas propiciando la interacción social entre iguales y dando como resultado el aumento de la autoestima.

En los últimos años han sido diversas las iniciativas que, desde el ámbito de las tecnologías digitales, han generado apoyos para compensar las dificultades que 
presentan las personas con TEA. Pero, ¿por qué las Tecnologías de la Información y la Comunicación (en adelante TIC) para las personas con TEA?

A continuación presentamos los principales motivos por los cuales las tecnologías digitales favorecen el desarrollo de las personas con TEA y en particular a los niños (Boser, Goodwin\&Wayland, 2014):

- Ofrecen un entorno y una situación controlable, son un interlocutor altamente predecible que ofrece contingencias perfectas y comprensibles: pulsando la misma tecla se obtiene siempre los mismos resultados.

- Presentan una estimulación multisensorial, fundamentalmente visual; ya que existe evidencia de que las personas con TEA perciben y procesan mejor la información recibida por el canal visual que por el auditivo, por este motivo se les considera pensadores visuales. Las representaciones visuales son una ayuda para la comunicación pero también para el desarrollo de otras áreas como la estructuración del pensamiento y el propio aprendizaje.

- Eliminan las barreras espacio-temporales para la educación.

- Su capacidad de motivación y refuerzo es muy alta, favoreciendo la atención y reduciendo la frustración ante los errores.

- Favorecen o posibilitan el trabajo autónomo y el desarrollo de las capacidades de autocontrol, las tecnologías digitales se adaptan a las características de cada uno, favoreciendo ritmos de aprendizaje diferentes y una mayor individualización.

- Son un elemento de aprendizaje activo, donde destacan por su versatilidad, flexibilidad y adaptabilidad.

- Potencian los escenarios y entornos de aprendizaje interactivos. 
- Son un recurso didáctico capaz de simular situaciones y contextos reales complejos e inseguros para las personas con TEA pero a la vez necesarias de trabajar.

Es sabido que las personas con TEA tienen una afinidad natural por el trabajo con las tecnologías porque entre muchos aspectos, estas proporcionan un entorno controlado y una atención individualizada capaz de adaptarse a sus necesidades educativas, favoreciendo el trabajo autónomo. También, ayudan a los niños con TEA a estructurar y organizar la exploración e interacción del entorno, así como dar un margen de tiempo más amplio para identificar una situación y darle una respuesta adecuada.

En pocas palabras, las tecnologías digitales permiten generar instrumentos de intervención que apoyen a las personas con TEA en muchos de sus retos diarios a la vez que potencian la mejora de futras intervenciones.

Aportaciones como las de Tseng y Yi-Luen (2011) ayudan a conocer la importancia de la integración de las TIC en todo proceso de intervención. 
Tabla 2: Ventajas e inconvenientes entre las intervenciones con TIC y los diseños de intervención tradicionales

\begin{tabular}{ll}
\hline \multicolumn{1}{c}{ Tecnologías de la información y la comunicación (TIC) } \\
\hline \multicolumn{1}{c}{ Ventajas } & \multicolumn{1}{c}{ Inconvenientes } \\
\hline - Mayor precisión. & Necesidades de acceso al \\
- Uso efectivo de tiempo y dinero en la & ordenador. \\
administración. & el funcionamiento requerido por el \\
- No requieren una interacción social. & ordenador. \\
- Los datos se pueden archivar a largo plazo. \\
- Fácil comparación de datos entre diferentes \\
grupos. \\
- Múltiples estímulos para elegir. \\
- Fácil proceso de sesiones complicadas. \\
- Alta resistencia para desarrollar sesiones a \\
largo plazo.
\end{tabular}

\begin{tabular}{ll}
\hline \multicolumn{1}{c}{ Diseños Tradicionales } \\
\hline - Menos necesidades de acceso. & \multicolumn{1}{c}{ Inconvenientes } \\
\hline - Fácil de usar. & - Dificultad para almacenar datos \\
& durante largos periodos. \\
& - Dificultad para realizar \\
& comparaciones intragrupo. \\
& - Dificultad para procesar \\
& procedimientos complicados.
\end{tabular}

Nota. Extraído de Tseng \& Yi-Luen (2011).

En definitiva, las tecnologías digitales proporcionan un marco en el cual se parte de los puntos fuertes y capacidades, adaptándose al estilo y ritmo de los procesos de 
enseñanza-aprendizaje (E-A) de cada persona hecho que favorece la personalización de la acción educativa.

Pese al amplio abanico de posibilidades que ofrecen las tecnologías digitales a la comunidad autista y a los profesionales del ámbito, es esencial comprenderlas como un medio para llegar a un fin: mejorar la calidad de vida de estas personas (Spiel, Frauenberger \& Fitzpatrick, 2017). Para ello, consideramos esencial que los propios usuarios, sus familiares y los profesionales se involucren en los procesos de diseño y evaluación de dicha tecnología, asumiendo un rol de co-diseñadores (Parsons, et al., 2016). Realmente, en ellos reside la clave para explotar los avances tecnológicos con el fin de ofrecer mejores respuestas a sus necesidades.

\section{Aplicaciones móviles para personas con TEA: ¿Cómo deben ser las Apps para personas autistas?}

Todo lo expuesto anteriormente ha provocado en los últimos años un interés masivo por desarrollar todo tipo de software y Apps especializadas para el uso de las personas con TEA. Es evidente que el auge de la creación de esta tipología de recursos tecnológicos viene dado por la popularidad de los dispositivos móviles inteligentes como las tabletas y los teléfonos inteligentes.

Desarrollarse y formarse como ciudadano en el contexto de una sociedad globalizada, dinámica e inestable como la nuestra supone un reto para las personas con TEA. Por este motivo el uso de las tecnologías digitales y en concreto de los dispositivos móviles resulta una oportunidad para contribuir a la mejora de su inclusión social permitiéndoles acceder, crear y distribuir conocimiento libremente.

Este tipo de dispositivos presentan ciertas condiciones que resultan de gran interés para la intervención educativa de las personas con necesidades de aprendizaje 
específicas y en concreto de las personas con TEA. Diversos estudios destacan el uso de Apps educativas como un recurso eficiente sobre todo para la intervención en el área socio-comunicativa, trabajando así las dificultades presentes en el lenguaje y las competencias comunicativas. En esta área también destacan por su vinculación con el uso de los Sistemas Aumentativos y Alternativos de Comunicación (SAAC).

Evidenciadas las aportaciones de este tipo de dispositivos, pasamos a concretar qué características lo hacen posible. Primeramente, como ya se ha abordado con anterioridad en este artículo, por la habilidad natural de las personas con TEA para procesar y comprender mejor a través de la información y las claves visuales en, por ejemplo, una tableta. En segundo lugar, por la alta disponibilidad de esta tecnología considerada casi universal. Y por último por sus aspectos técnicos:

- Interfaz visual y clara.

- Teclas tipo pictogramas y no de texto.

- Funcionamiento lógico.

- Libre de estímulos distractores.

A partir de su experiencia Boyd, Barnett y More (2015) presentan cinco factores esenciales a tener en cuenta en el momento de seleccionar una App para la intervención con niños con TEA:

- La capacidad de personalizar la aplicación.

- Las habilidades motoras que el alumno necesita para operar correctamente con el sistema.

- Los recursos y el tiempo necesario para el desarrollo de la intervención educativa.

- La investigación o las prácticas basadas en la evidencia vinculadas al diseño, desarrollo y evaluación de la aplicación. 
- El coste de usar el dispositivo y la aplicación específica.

Como recomendación a añadir a estos cinco factores, consideramos interesante conocer si en el proceso de creación y diseño de la App han participado personas con TEA.

En definitiva, las Apps educativas para personas con TEA deben tener desarrollos sencillos e intuitivos para su utilización, deben estar libres de estímulos distractores y basarse en un funcionamiento acorde con las metodologías específicas de intervención con personas con TEA: uso de apoyos visuales para la realización de las tareas, aprendizaje sin error, actividades bien definidas y con opciones de configuración que permitan ajustarlas en la medida de lo posible a cada persona.

Por todo esto, podemos decir que existen un gran número de Apps pero también que no existen dos perfiles de TEA iguales.De ahí, la necesidad de seleccionar cuidadosamente las Apps con las que se intervendrá. Con este fin se encuentran disponibles diferentes repositorios de Apps para personas con TEA, categorizándolas según: (1) el área de intervención, (2) plataforma compatible, (3) edad de los usuarios y (4) si se han desarrollado según evidencias científicas (AppyAutism, 2014; AutismSpeaks, 2013).

\section{Conclusiones y nuevos retos}

Aunque la sociedad digital ha impregnado también los procesos educativos y las diferentes instituciones educativas, no podemos garantizar que los procesos de digitalización aseguren por si mismos estrategias de inclusión educativa que consideren la diversidad de sujetos a los que debemos atender des del punto de vista educativo. 
A continuación concretamos una serie de conclusiones a partir de los diferentes aspectos tratados a lo largo de este artículo.

Las tecnologías digitales han supuesto un importante avance respecto a la superación de metodologías tradicionales en todo aquello relativo a la adaptación de los distintos estilos y ritmos de aprendizaje (Tseng \& Yi-Luen, 2011). En este sentido, utilizar este tipo de herramientas con las personas con TEA permite ampliar y mejorar sus posibilidades de desarrollo, así como optimiza su proceso de aprendizaje y formación. Actualmente, existe una gran cantidad de Apps para la atención educativa de las personas con TEA y para la contribución a la mejora su calidad de vida. Dentro de este escenario de posibilidades digitales, la dificultad reside en acceder a ellas, seleccionarlas, conocerlas y hacer un uso adecuado para cada perfil de usuario, dada su heterogeneidad. Ante la falta de un sistema acordado u oficial de clasificación consideramos importante establecer, primero, una serie de criterios de búsqueda para esta tipología de Apps. Segundo, determinar un conjunto de criterios pedagógicos y de diseño visual e interactivo para evaluar su calidad y adaptación al desarrollo de las personas con TEA (Crescenzi-Lanna \& Grané-Oró, 2016; Boyd, et al., 2015).

Todas estas observaciones se relacionan también con el papel fundamental de los profesionales de la educación y la familia en la búsqueda y elección de las "mejores" tecnologías digitales, consideradas como potenciales recursos para el aprendizaje de este colectivo. Hay que mencionar, además la necesidad de formar a los educadores y desarrolladores de estas tecnologías en este aspecto así como en competencia digital. También, el uso de Apps educativas favorece la creación de un espacio colaborativo entre los diferentes miembros de la comunidad educativa para apoyar el proceso de aprendizaje de las personas con TEA. 
Para concluir, las evidencias de las diferentes investigaciones manifiestan que las tecnologías digitales constituyen una herramienta y un recurso muy importante desde la perspectiva de la mejora de los procesos educativos de los alumnos con TEA (Boser et al., 2014). No obstante hemos de ser conscientes que en los procesos sociales y educativos, lo más importante es orientar nuestro discurso y práctica educativa a los procesos didácticos, más que a las herramientas tecnológicas en sí mismas. Solo así, estaremos contribuyendo a la mejora de la inclusión digital de estas personas, no solo desde una perspectiva educativa sino también personal y social.

\section{Referencias}

AppyAutism. (2014). AppyAutism. Recuperado de: http://www.appyautism.com/. Asociación Americana de Psiquiatría (APA). (2013). Guía de consulta de los criterios diagnósticos del DSM V. Arlington, VA: American PsychiatricPublications. Autism Speaks. (2013). Autism apps. Recuperado de: http://www.autismspeaks.org/autism-apps/

Boser, K., Goodwin, M. \& Wayland, S. (Eds.). (2014). Technology tools for students with autism: Innovations that enhance independence and learning. London: Brookes Publishing.

Boyd, T., Barnett, J. \& More, C. (2015). Evaluating iPad technology for enhancing communication skills of children with Autism Spectrum Disorders. Intervention in School and Clinic, (51),19-27. doi: 10.1177/1053451215577476.

Centers for Disease Control and Prevention, CDC. (2015).AutismSpectrumDisorder.Data \&Statistics.Recuperado de: https://www.cdc.gov/ncbddd/autism/data.html/. 
Cisco. (2016). Informe Anual de Seguridad. Recuperado de http://www.cisco.com/c/dam/m/es_es/internet-of-everythingioe/iac/assets/pdfs/security/cisco_2016_asr_011116_es-es.pdf

Crescenzi-Lanna, L. \& Grané-Oró, M. (2016). Análisis del diseño interactivo de las mejores apps educativas para niños de cero a ocho años. Comunicar, 24(46), 77-85. https://doi.org/10.3916/C46-2016-08

Ditrendia. (2016). Informe Mobile en España y en el Mundo 2016. Recuperado de http://www.amic.media/media/files/file_352_1050.pdf

Fundación Orange. (2014). eEspaña 2014. Informe anual sobre el desarrollo de la sociedad de la información en España. Madrid: Fundación Orange.

Recuperado de

http://www.proyectosfundacionorange.es/docs/eE2014/Informe_eE2014.pdf

Gisbert, M., Prats, M.A. \& Cabrera, N. (2015).Aprenentatgemòbil. Com incorporar elsdispositiusmòbils a l'aprenentatge?. Barcelona: Fundació Bofill. Informes breus, 58. Recuperado de:

http://www.fbofill.cat/sites/default/files/InformeBreu58.pdf

Hansen, S., Schendel, D. \&Parner E. (2015).Explaining the increase in the prevalence of autism spectrum disorders. The proportion attributable to changes in reporting practices.JAMA Pediatrics. 169(1), 56-62. doi:

10.1001/jamapediatrics.2014.1893

Kientz, J., Goodwin, M., Hayes, G. \&Abowd, G. (2013).Interactive technologies for autism. Synthesis Lectures on Assistive, Rehabilitative and Health-Preserving Technologies, 2(2), 1-177. doi: 10.2200/S00533ED1V01Y201309ARH004 
Lázaro, J.L., Estebanell, M. \& Tedesco, J.C. (2015). Inclusion and Social Cohesion in a Digital Society.RUSC. Universities and Knowledge Society Journal, 12(2).doi: http://dx.doi.org/10.7238/rusc.v12i2.2459

Monclús, A. \&Saban, C. (2012). La inclusión, la desigualdad y la brecha digital, como problemas y retos para las nuevas tecnologías de la información y de la comunicación. Revista Iberoamericana de Educación, 60, 2, 1-10. Recuperado de: http://goo.gl/g0wAzG

Parsons, S., Leonard, A. \&MitchelL, C. (2006). Virtual Environments for Social SkillsTraining: CommentsfromTwo Adolescents withAutisticSpectrumDisorder. Computers\&Education, 47(2),186-206. Recuperado de: http://www.sciencedirect.com/science/article/pii/S0360131504001460/.

Parsons, S., Yuill, N., Good, J., Brosnan, M., Austin, L., Singleton, C. \&Bossavit, B. (2016). What Technology for Autism Needs to be Invented? Idea Generation from the Autism Community via the ASCmel.T. App. En K. Miesenberger, C. Bühler y P. Penaz (Eds.) Computers Helping People with Special Needs, 343350. Springer. doi: 10.1007/978-3-319-41267-2_49

Picard, G. \& Pickard, V. (2017). Essential Principles for Contemporary Media and Communications Policymaking. Reuters Institute for the Study of Journalism: University of Oxford. Recuperado de: http://reutersinstitute.politics.ox.ac.uk/publication/essential-principlescontemporary-media-and-communications-policymaking

Rivière, Á. (2002). IDEA: Inventario de espectro autista. Buenos Aires: Fundec.

Rivoir, A. (2009). Innovación para la inclusión digital. El Plan Ceibal en Uruguay. Mediaciones Sociales, (4), 299-328. Recuperado de: 
http://europa.sim.ucm.es/compludoc/AA?articulold=746753\&amp;donde=castell ano\&amp;zfr $=0 /$.

Spiel, K., Frauenberger, C. \& Fitzpatrick, G. (2017).Experiences of autistic children with technologies. International Journal of Child-Computer Interaction, 11, 50-61. doi: 10.1016/j.jjcci.2016.10.007

Telefónica (2016). La Sociedad de la Información en España 2016. Madrid: Fundación Telefónica. Recuperado de: www.fundaciontelefonica.com/publicaciones/.

Tseng, R. Y. \&Yi-Luen, E. (2011). Therole of Informationand Computer Technology for childrenwithAutismSpectrumDisorderandthe Facial expressionWonderland (FeW). International Journal of Computational Models andAlgorithms in Medicine, 2, 23-41. doi: 10.4018/jcmam.2011040102.

UNESCO (2014). Informe sobre el uso de las tecnologías de información y comunicación (TIC) en la educación para personas con discapacidad. Informe Mundial. Lima: Unesco. Recuperado de:

http://unesdoc.unesco.org/images/0021/002163/216382s.pdf

\section{Formato de citación}

Sanromà-Giménez M., Lázaro-Cantabrana J. y Gisbert-Cervera M. (2017). La tecnologíamóvil. Una herramienta para la mejora de la inclusión digital de las personas con TEA. Psicología, Conocimiento y Sociedad, 7(2), 227-251. Disponible en: http://revista.psico.edu.uy/ 